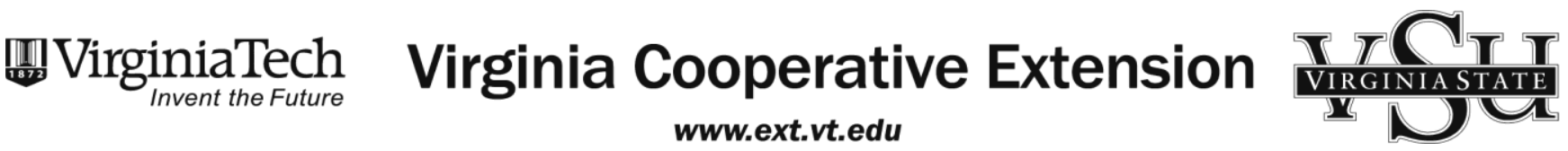

\section{Velvet Ants}

Hymentoptera: Mutillidae

Theresa A. Dellinger and Eric Day, Department of Entomology, Virginia Tech

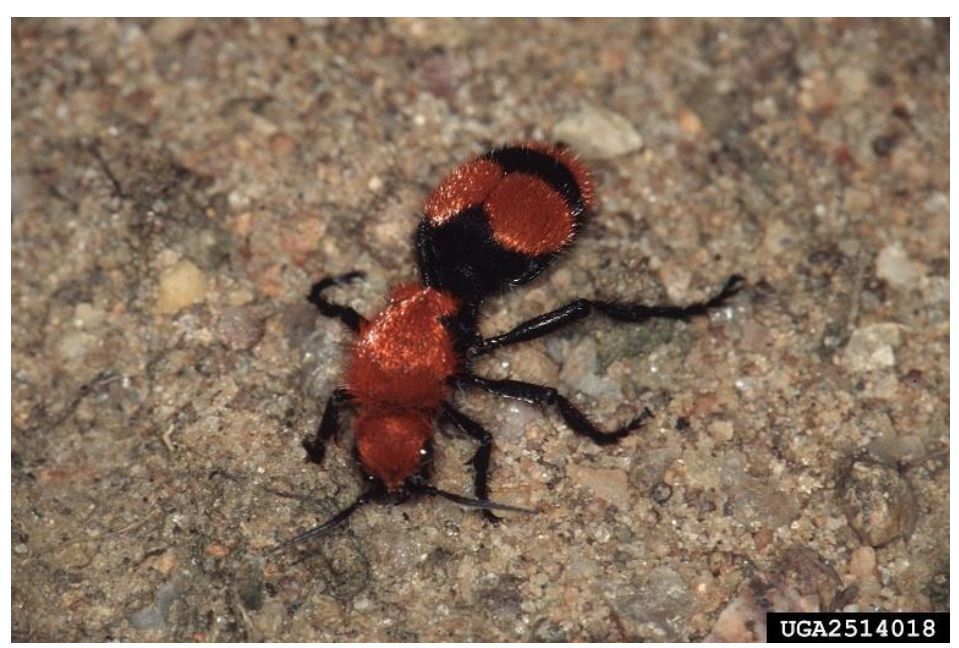

Female velvet ant

[Jerry A. Payne,USDA-ARS, Bugwood.org]
Description Velvet ants are medium-sized insects that resemble large hairy ants but are actually wasps. Their bodies are densely clothed with hair that gives them a velvety appearance. Females lack wings; males have wings but are rarely seen. The velvet ants found in Virginia are brightly colored red and black, sometimes with thinner bands of white or gray at the tip of the abdomen.

Damage Velvet ants are not considered pests. Adult females are not particularly aggressive and sometimes make a squeaking or chirping noise when handled. However, picking up a velvet ant is unadvised as they can also inflict an extremely painful sting. Velvet ants stings should be treated as bee stings.

Habitat/Distribution Velvet ants are not uncommon insects but often go unnoticed. They are usually seen in open fields and pastures with bare or sandy soil where ground-nesting bees and wasps are found.

Control Individual velvet ants can be killed with an aerosol spray or a fly swatter. Because host nests containing developing velvet ants cannot be readily identified, no control measures are suggested for immature velvet ants.

Life cycle Velvet ants have a complete life cycle consisting of egg, larval, pupal and adult stages. A female velvet ant usually invades a nest of ground-nesting wasps or bees to lay her eggs among the mature host larvae and pupae. Sometimes the nests of bumble bees are attacked. Velvet ant larvae parasitize and eat the host larvae and pupa before pupating in the host nest. Adult velvet ants live solitary lives and do not form hives or live in groups. They feed on flower nectar and pollen.

Interesting Facts Velvet ants are sometimes called "cow killers" because of their extremely painful sting, although it's very unlikely that their venom could actually kill a cow. 